\title{
Research Advances of Typical Two Dimensional Layered Thermoelectric Materials
}

\author{
Haihua HUANG, Xiaofeng FAN ${ }^{\star}$
}

Key Laboratory of Automobile Materials (Jilin University), Ministry of Education, and College of Materials Science and Engineering, Jilin University, Changchun, 130012, China

\section{*Corresponding Author: Xiaofeng FAN, Email: xffan@jlu.edu.cn}

\begin{abstract}
:
Thermoelectric technologies have caught our intense attention due to their ability of heat conversion into electricity. The considerable efforts have been taken to develop and enhance thermoelectric properties of materials over the past several decades. Recently, twodimensional layered materials are making the promise for potential applications of thermoelectric devices because of the excellent physical and structural properties. Here, a comprehensive coverage about recent progresses in thermoelectric properties of typical two dimensional (2D) layered materials, including the theoretical and experimental results, is provided. Moreover, the potential applications of $2 \mathrm{D}$ thermoelectric materials are also involved. These results indicate that the development of $2 \mathrm{D}$ thermoelectric materials take a key role in the flexible electronic devices with thermoelectric technologies.
\end{abstract}

Keywords: thermoelectric properties; transport properties; $2 D$ layered materials; figure of merit, first-principle calculations

\section{Introduction}

Thermoelectric (TE) conversion technology, which is a kind of green energy technology and can convert waste heat into electricity, has been considered to be an alternative for relieving energy shortage. TE devices have been used in different directions, including solid state cooling and deep space exploration $^{[1-2]}$, and also shown the high potential in other areas, such as sensors and power generators ${ }^{[3-5]}$. These make TE materials a kind of important compounds which support the development of sustainable energies and technologies with high efficiency. The performance of TE materials usually is characterized by the figure of merit $Z T$ which is a dimensionless parameter and expressed as $^{[6]}$, $Z T=S^{2} \sigma T / \kappa$, where $S$ is the Seebeck coefficient and $\sigma$ is the electrical conductivity. $\mathcal{\kappa}$ presents the thermal conductivity and $T$ is the abbreviation of absolute temperature. There are two parts for the thermal conductivity $\kappa$ which are including the electronic $\left(\kappa_{e}\right)$ and lattice contributions $\left(\kappa_{l}\right)$. These parameters are coupled to each other and strongly depend on the electronic structure and carrier concentration of the materials. There are usually two approaches to improve the TE performance. One way is to decrease the thermal conductivities $\kappa_{e}$ and $\kappa_{p}$, and the other is to increase the power factor $S^{2} \sigma$. In the past few decades, the TE field has made great progress, including various strategies to increase the power factor and reduce the thermal conductivity. In the TE materials family there are a lot of candidates which have been reported to achieve an obvious enhancement for the value of $Z T^{[7-12]}$. Among these kinds of strategies, lowdimensional material system has become a focus with the development of the nanotechnologies and has been applied to the thermoelectrics. Moreover, TE devices on the basis of flexible materials with large size have shown the great potential with the rapid emergence of the concept of the "Internet of Things (IoT)" ${ }^{[13]}$, especially in flexible wearable devices. It has many advantages, such as long lifetime and maintenance-free. In addition, the devices of TE energy conversion don't give rise to the vibrations or noises, while they are suitable at moderate temperatures ${ }^{[14]}$. Thus, TE properties of low-dimensional materials have attracted much attention ${ }^{[15-16]}$.

To date, the research of TE materials has shown the prominent progress with the enhancement of $Z T$ value, due to the inspirations by the design of low-dimensional structures, mainly two-dimensional (2D) structures for TE proposed in $1990 s^{[17]}$. The core idea behind with lowdimensional structures to enhance the TE performance is to introduce the dramatic differences in the electronic density of states (DOS) near the Fermi level. In this way, the electrical conductivity, thermal conductivity and Seebeck coefficient can be modulated quasi-independently by 
quantum-confinement effects from three-dimensional to two-dimensional ${ }^{[18]}$. With the development of preparation technologies, A series of 2D materials have been fabricated successfully ${ }^{[19-28]}$. It is possible to develop new lowdimensional TE materials from the perspective of twodimensional materials.

Initially, the research on 2D TE materials was mainly focused on quantum well and super-lattice thin film materials ${ }^{[29-31]}$, such as GaAs/AlAs, $\mathrm{Bi}_{2} \mathrm{Te}_{3} / \mathrm{Sb}_{2} \mathrm{Te}_{3}$ and $\mathrm{SrTiO}_{3} / \mathrm{SrTi}_{0.8} \mathrm{Nb}_{0.3} \mathrm{O}_{3}$. To date, although there have been some summaries on the related researches about 2D TE materials and the progress of the TE devices based on $2 \mathrm{D}$ materials ${ }^{[14-15,32]}$, an interesting review about new layered $2 \mathrm{D}$ materials with different structure types for the potential applications in TE field is still missing. In this short review, we are focused on the progress in the new $2 \mathrm{D}$ thermoelectric materials theoretically and experimentally, such as group IV-VI layered materials, III-VI layered materials, group IV-V compounds and transition metal dichalcogenides (TMDs). We review the new TE properties in these types of $2 \mathrm{D}$ structures and also provide some insight for designing the new layered TE materials in the future.

\section{TE Properties of typical 2D TE Materials}

\subsection{Group IV-VI two-dimensional layered materials}

As a typical one of layered group IV-VI materials, SnSe with the special atomic structure has the excellent electronic transport and thus has attracted wide attention. It possesses a orthorhombic structure with layered atomic arrangement under space group of $P n m a^{[21]}$. In figure 1a, its structure is along the $b$-axis in the perspective view. Due to this special layered structure, the carriers' mobilities are highly anisotropic along different transport directions. As depicted in figure $1 \mathrm{~b}$, the carrier's mobility at room temperature is $250 \mathrm{~cm}^{2} \mathrm{~V}^{-1} \mathrm{~s}^{-1}$ along the $b$-axis. This value is 10 times as high as that of $a$-axis which is outside the plane. Due to the folded plane, the mobility of carrier along $c$-axis is also obvious different, compared with that along the $a$-axis. On account of the lone electron pairs of the $\mathrm{Sn}^{2+}$ atoms, SnSe also exhibits ultralow thermal conductivity of $0.34 \mathrm{~W} \mathrm{~m}^{-1} \mathrm{~K}^{-1}$ at $773 \mathrm{~K}$ along the $b$-axis. Combined superior mobility and ultralow thermal conductivity, the $\mathrm{SnSe}$ shows a high $Z T$ value along $b$-axis at $773 \mathrm{~K}$ for p-type which is 2.3 , as shown in figurelc. This value is a higher value among the recent experimental reports in the published literatures. Moreover, because of the layers' weak van der Waals interaction, the single-layer with inlayer strong covalent bonds can be fabricated from the bulk SnSe. The cleavage energy for splitting layers is about 200 meV/atom theoretically ${ }^{[33]}$. The calculation results show that the single-layer SnSe is with an energy gap of $1.28 \mathrm{eV}$ and possesses the thermodynamic stability ${ }^{[34-35]}$. It has been reported that the $Z T$ value of single-layer SnSe at $773 \mathrm{~K}$ could reach to 3.27. Furthermore, it has been demonstrated that SnSe with orthorhombic Pnma symmetry (denoted as a-SnSe) has a phase transition at $796 \mathrm{~K}$ and becomes to be the -SnSe with orthorhombic structure under space group $\mathrm{Cmcm}^{[36]}$. These suggested that the new phases may be realized by changing growth temperature. Since then, the electronic and TE properties of many potential new phases (the polymorph structures of SnSe are shown in figureld) have been researched ${ }^{[37]}$. The theoretical results show that the $\beta$-SnSe phase has a $Z T$ value of 2.06 at room temperature ${ }^{[37]}$. (a)

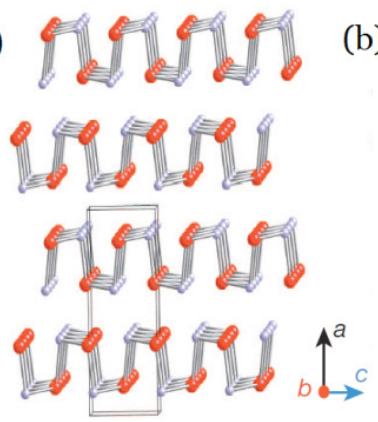

(d)

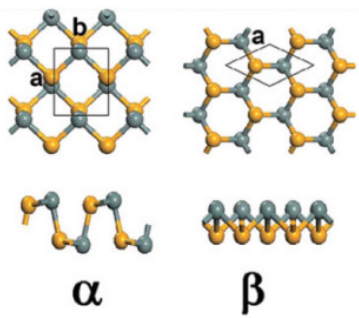

(b)

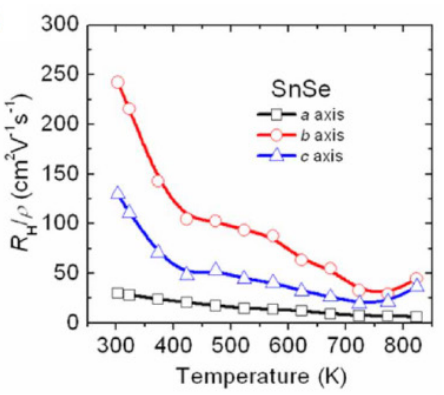

(c)

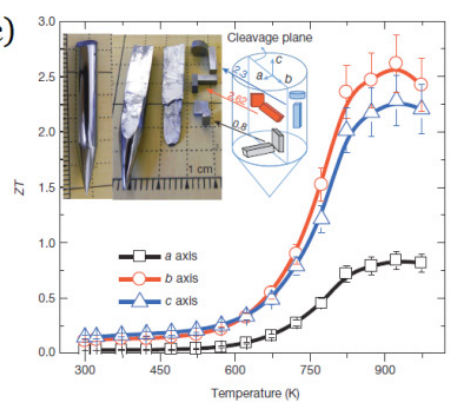

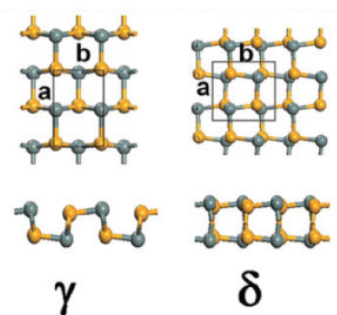

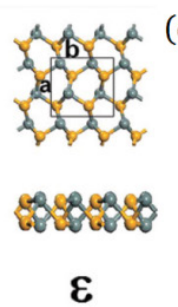

(e)

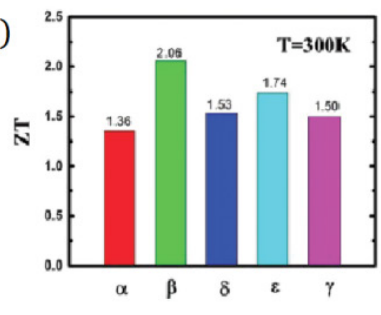

Figure 1 (a) Crystal structure of SnSe. (b) $R_{\mathrm{H}} / \rho$ ( $\rho$, electrical resistivity) of SnSe along different axial directions. (c) $Z T$ values of SnSe along different directions. Reproduced with permission from ref. 21. Copyright 2014, NPG. (d) Geometric structures of $\alpha-, \beta-, \gamma-, \delta$ - and $\varepsilon$-SnSe monolayers. Gray atoms denote $\mathrm{Sn}$ atoms and yellow atoms denote Se atoms. (e) ZT values of different monolayer SnSe phases at 300 K. Reproduced with permission from ref.37. Copyright 2017, RSC. 
These results above have sparked interest in exploring other group IV-VI two-dimensional materials ${ }^{[38-42]}$, such as GeSe, SnS, GeS, $\mathrm{SnS}_{2}, \mathrm{SnSe}_{2}, \mathrm{SiS}$ and SiSe. Theoretical calculations have revealed that group IV-VI compounds with folded structure could exhibit high Seebeck coefficient, relative low thermal conductivity and had the potential toward TE applications ${ }^{[43]}$. The thermal transport property of group IV-VI single-layer materials (GeS, GeSe, $\mathrm{SnS}$ and $\mathrm{SnSe}$ ) has been investigated by first-principles calculations ${ }^{[42]}$. The average thermal conductivity calculated is $6.38 \mathrm{Wm}^{-1} \mathrm{~K}^{-1}, 5.23 \mathrm{Wm}^{-1} \mathrm{~K}^{-1}, 3.08 \mathrm{Wm}^{-1} \mathrm{~K}^{-1}$ and $2.77 \mathrm{Wm}^{-}$ ${ }^{1} \mathrm{~K}^{-1}$, respectively. Among these IV-VI materials studied, GeS has the largest Seebeck coefficient $\left(2810 \mathrm{VK}^{-1}\right)$ at room temperature ${ }^{[41]}$. Compared with their bulk materials, the optimal ZT values of single-layer GeSe, GeS and $\mathrm{SnS}$ are high and 1.99, 1.85 and 1.88, respectively. Therefore, these materials are expected to have the potential applications in TE devices. In addition, as typical group IV-VI materials, SiSe and SiS possess high Seebeck coefficient arising from the sharp DOS near Fermi level ${ }^{[44]}$. The calculation results about the power factor indicate that the peak values of the p-type and $n$-type are $16 \mathrm{mWm}^{-2} \mathrm{~K}^{-2}$ and $11 \mathrm{mWm}^{-2} \mathrm{~K}^{-2}$, respectively. these values are similar to the experimental report on the state-of-the-art material SnSe with the layered structure ${ }^{[35]}$.

\subsection{Group III-VI two-dimensional layered materials}

As the potential TE materials, the compounds which belong to III-VI group have aroused much attention during the past few years. These materials, including InSe, $\mathrm{GaSe}, \mathrm{GaS}$ and $\mathrm{Tl}_{2} \mathrm{O}$, have been extensively studied in the TE field ${ }^{[45-47]}$. Taking InSe as an example, with the stoichiometric ratio of $1: 1$, it has the typical structure of group III-VI layered materials shown in figure $2 \mathrm{a}$. It has been also noticed that In-Se system is complex and there are other different stoichiometric ratios ${ }^{[48]}$, such as $\operatorname{In}_{4} \mathrm{Se}_{3}{ }^{[49]}$, $\mathrm{In}_{2} \mathrm{Se}_{3}{ }^{[50]}, \mathrm{InSe}^{[51]}, \mathrm{In}_{6} \mathrm{Se}_{7}{ }^{[52]}$ and $\mathrm{In}_{3} \mathrm{Se}_{4}{ }^{[53]}$. In addition, with the same stoichiometric ratio, different phases and crystal structures may coexist. Geim et al. ${ }^{[54]}$ reported that the carrier's mobility of the thin InSe was very high. It exceeds $10^{3} \mathrm{~cm}^{2} \mathrm{~V}^{-1} \mathrm{~s}^{-1}$ at RT and is about $10^{4} \mathrm{~cm}^{2} \mathrm{~V}^{-1} \mathrm{~s}^{-1}$ at liquidhelium temperature. Moreover, experimental studies show that the thermal conductivity of bulk $\operatorname{In}_{4} \mathrm{Se}_{2.35}$ is very low along the $b$ - $c$ plane $\left(1.2 \mathrm{Wm}^{-1} \mathrm{~K}^{-1}\right.$ at $\left.300 \mathrm{~K}\right)$, and it decreases with increasing temperature $\left(0.74 \mathrm{Wm}^{-1} \mathrm{~K}^{-1} \text { at } 705 \mathrm{~K}\right)^{[55]}$.

Based on the analysis of the electronic structure of IIIVI compounds with single-layer and multi-layer, it is found that the valence band of these materials have a "Mexican hat" dispersion ${ }^{[56-59]}$, which can increase the density of states near the band edge (as seen in figure $2 b$ ), and further increases the Seebeck coefficient. Single- and multi-layer materials of InSe, GaSe and GaS have been successfully fabricated experimentally ${ }^{[60-63]}$. Subsequently, the electronic and TE properties of these materials from single-layer to multi-layer have been researched extensively. The results show that the power factor increases, following the material's thickness decreases ${ }^{[64-65]}$. As an example, the maximum power factor of single-layer InSe reaches to
$16 \mathrm{mWm}^{-2} \mathrm{~K}^{-2}$. The TE performance of single-layer InSe has been also investigated theoretically through using mechanical strain. Under the strain, the energy bands will converge and thus lead to an increase of power factor, while the thermal conductivity is also found to decrease ${ }^{[66]}$.

Some works have revealed that the sharp conduction band edge with high DOS due to the quantum confinement could lead to the enhancement of the Seebeck coefficient ${ }^{[67]}$. The DOS and the information of electronic wave functions at conduction band maximum CBM for 9-layers and 36-layers InSe films are shown in figure 2c. With the combination of theoretical simulations and experimental tests, it is found that the power factor is increased obviously, by following the thickness of InSe sample decreases, especially less than the thermal wavelength (seen in figure $2 \mathrm{~d}$ ). This may provide a guideline for further optimizing the TE properties of 2D layered structures.

\subsection{Group IV-V two-dimensional layered materials}

With the emergence of group IV and group V 2D layered materials, including graphene, silicene, germanene, phosphorene and antimonene, group IV-V 2D compound materials have been also explored ${ }^{[68-70]}$. Group IV-V compounds are known to form the layered structures. A few structures have been synthesized at high pressure ${ }^{[71]}$, such as $\mathrm{SiP}, \mathrm{SiAs}, \mathrm{GeAs}$ and GeP. The layered crystal structure with space group $C 2 / m$ is shown in figure $3 a$.

As a typical one, GeAs with anisotropic atomic structure has demonstrated highly anisotropic properties of transport ${ }^{[72]}$. As shown in figure $3 b$, the resistivity perpendicular to the layers is very high due to large distance of layers with weak coupling. It is 2 orders of magnitude higher than that along the layers at $300 \mathrm{~K}$. The TE properties of Sn-doped GeAs have been studied. The results revealed that at high temperature, $\mathrm{Sn}$-doped GeAs exhibited superior transport properties, compared to the pristine GeAs. It has shown that $\mathrm{Sn}$-doped GeAs possesses a remarkable $Z T$ with a maximum of 0.35 at $660 \mathrm{~K}$ (as shown in figure $3 \mathrm{c})^{[72]}$. On the $\mathrm{Ge}-\mathrm{As}$ phase diagram, there are another compound $\mathrm{GeAs}_{2}$ with an orthorhombic crystal structure (in figure $3 \mathrm{~d})^{[73]}$. It exhibits the strong interlayer coupling due to its special stacking of layers with small layers' spacing along vertical direction (about $1 \AA$ ). The power factor of the $n$-type $\mathrm{GeAs}_{2}$ along the $b$-axis can reach to $4.2 \mathrm{~mW} / \mathrm{mK}^{2}$ This are comparable to the value $\left(4 \mathrm{~mW} / \mathrm{mK}^{2}\right)$ of SnSe ${ }^{[74]}$. Monolayer $\mathrm{GeAs}_{2}$ has complex bonding characteristic, and this is similar to the structural characteristic of SnSe. The calculated in-plane lattice thermal conductivities is 0.68 $\mathrm{Wm}^{-1} \mathrm{~K}^{-1}$ along the $a$-axis and $6.03 \mathrm{Wm}^{-1} \mathrm{~K}^{-1}$ along $c$-axis at RT, respectively ${ }^{[75]}$. These research achievements suggest that the group IV-V compound materials are the promising candidates for environmentally friendly TE applications.

\subsection{TMD materials}

It has been well known, TMDs are with typical layered stacking and the coupling of layers is by van der Waals interaction. For the singer layer, there are usually two different structural types. One is the hexagonal structure (H-phase). The typical materials include Mo and $\mathrm{W}$ based 

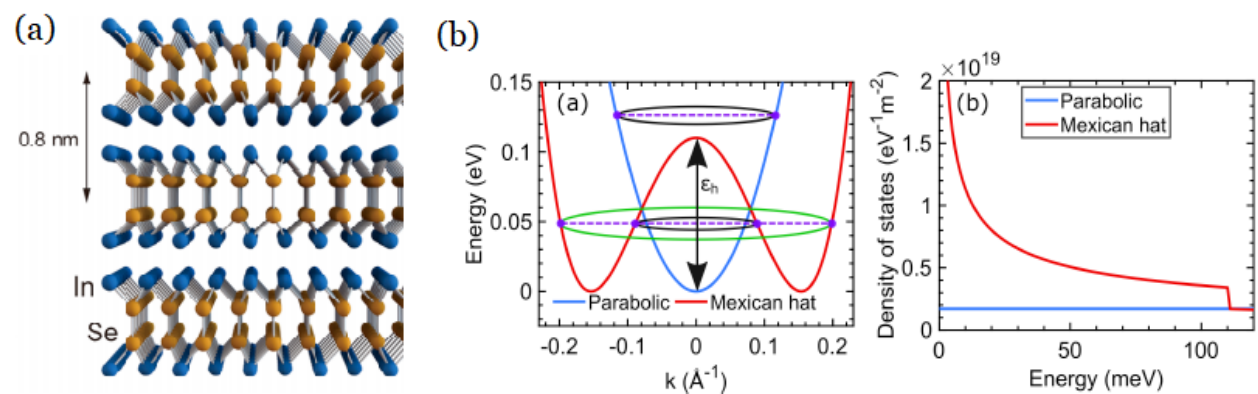

(c)

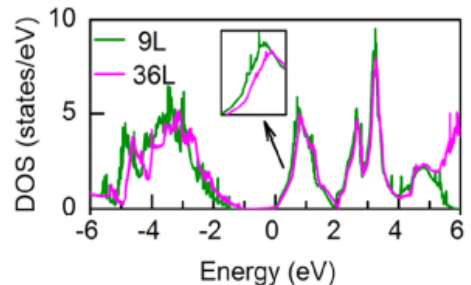

(d)

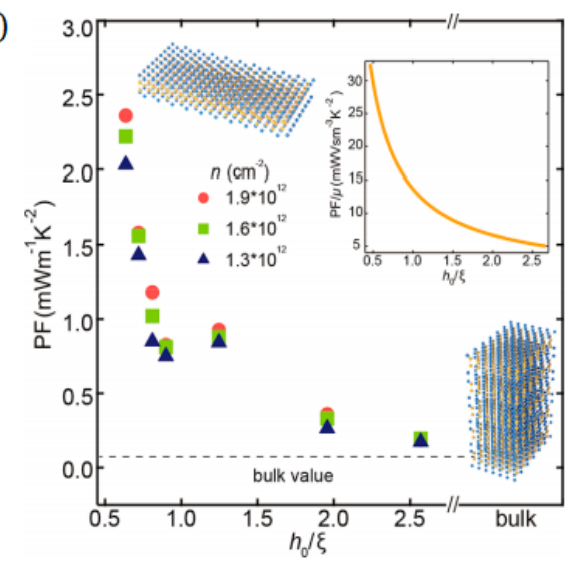

Figure 2 (a) Crystal structure of InSe. Reproduced with permission from ref. 67. Copyright 2018, ACS. (b) Comparison of a parabolic and Mexican hat dispersion and density of states of both. Reproduced with permission from ref. 59. Copyright 2019, APS. (c) Total density of states and planar-averaged squared magnitude of wave functions (which are from the states of conduction band maximum and plotted along the direction perpendicular to layers) of 9-layers and 36-layers InSe films (upper panel), (lower panel). (d) Power factor as functions of films' thickness with quantum confinement effect $\left(h_{0} / \xi\right)$ for the different carrier concentrations. ( $h_{0}$ is for quantum confinement of length, and $\xi$ is for heat wavelength). Reproduced with permission from ref. 67. Copyright 2018, ACS.

(a)

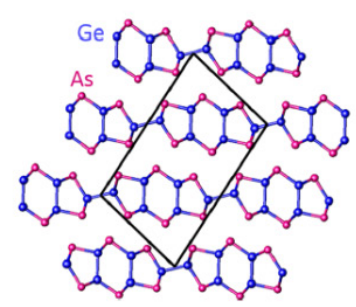

(d)

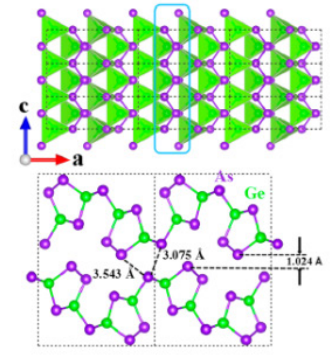

(b)

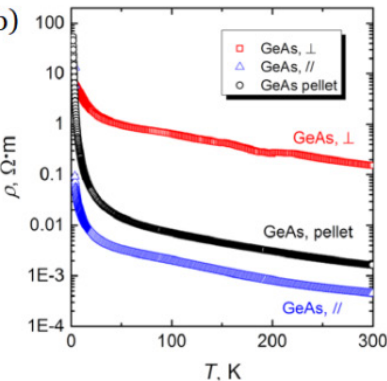

(e)

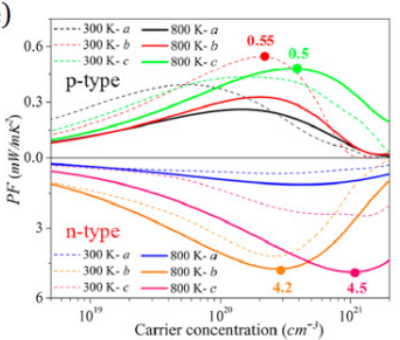

(c)

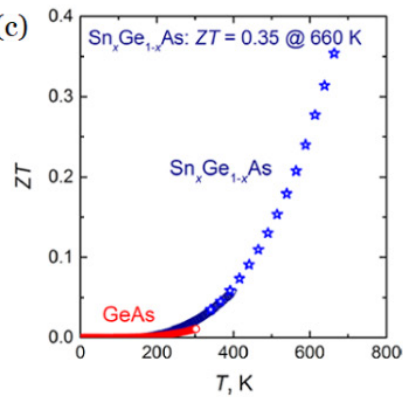

(f)

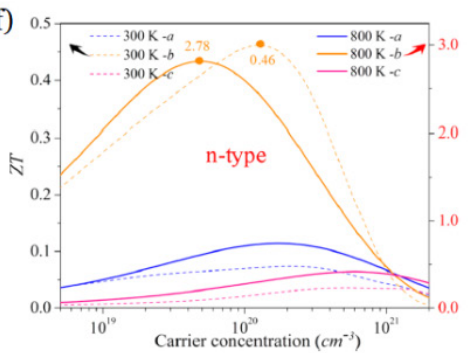

Figure 3 (a) Crystal structure of GeAs. (b) Resistivity of GeAs pellet and the GeAs crystal as functions of temperature. (c) ZT value as functions of temperature for $\mathrm{Sn}_{x} \mathrm{Ge}_{1-x}$ As pellet and GeAs. Reproduced with permission from ref. 72 . Copyright 2016, ACS. (d) Top view and side view of GeAs, atomic structure. (e) Power factors as functions of carrier concentration with different temperature for the $p$-type and $n$-type along different axes. (f) Calculated ZT value for the $n$-type GeAs. Reproduced with permission from ref. 73. Copyright 2017, ACS. 
dichalcogenides, such as $\mathrm{MX}_{2}(\mathrm{M}=\mathrm{Mo}, \mathrm{W}$, and $\mathrm{X}=\mathrm{S}, \mathrm{Se}, \mathrm{Te})$. The other one is the 1T-CdI type with trigonal structure ${ }^{[76]}$. In both structures, single layer of metal atoms is sandwiched between two chalcogen layers. Hexagonal structure is shown in figure 4a. They have been widely explored in various fields, such as electronics and optoelectronics, due to their diverse physical and chemical properties ${ }^{[77]}$. TMDs have been also researched as candidate materials for TE devices because of their high Seebeck coefficients and low thermal conductivities ${ }^{[78-82]}$.

The TE properties of 2D TMDs, such as $\mathrm{MoS}_{2}$, have been studied by both theoretical calculations and experimental works. The electronic structure has a significant layered effect for TMDs. For an example, their indirect band gap in multi-layers and bulk changes into direct band gap in their monolayer limit ${ }^{[83]}$. The band structure exhibits obvious changes by following the increase of atomic layer's number from monolayer to multilayer ${ }^{[84-86]}$. The TE transport properties of few-layer $\mathrm{MoS}_{2}$ have been reported ${ }^{[87]}$. As shown in figure $4 \mathrm{~b}$ and $4 \mathrm{c}$, the electrical conductivities, Seebeck coefficient and power factor have been measured ${ }^{[87]}$. The power factor increases correspondingly, by following the increase of applied gate voltage $V_{\mathrm{g}}$ on account of increasing electrical conductivity. Apparently, the device with double layer $\mathrm{MoS}_{2}$ has a large effective mobility of $64 \mathrm{~cm}^{2} \mathrm{~V}^{-1} \mathrm{~s}^{-1}$ with a maximum power factor of $8.5 \mathrm{mWm}^{-1} \mathrm{~K}^{-2}$ at RT. The value of power factor is the highest one among that measured in semiconducting TMDs as the TE materials. The enhancement of transport performance is not only due to the quantum-confinement effect but also due to their valley degeneracies and large effective masses. Similar behavior was also found in other TMDs, such as $\mathrm{MoSe}_{2}, \mathrm{WS}_{2}$, and $\mathrm{WSe}_{2}^{[88-89]}$.

The $2 \mathrm{D}$ materials, such as $\mathrm{TiX}_{2}, \mathrm{Zr} \mathrm{X}$, and $\mathrm{HfX}_{2}$, are typically with $\mathrm{CdI}_{2}$ type. They have attracted much attention because of their lower lattice thermal conductivity than those of $\mathrm{W}$ and Mo based TMDs. The lower thermal conductivity is originated mainly from the strong hybridization between low lying optical phonon modes and acoustic phonon modes ${ }^{[00-91]}$. For instance, the theoretical calculation results indicate that the lattice thermal conductivity at $\mathrm{RT}$ are $1.2 \mathrm{Wm}^{-1} \mathrm{~K}^{-1}$ for $\mathrm{ZrSe}_{2}$ and $1.8 \mathrm{Wm}^{-1} \mathrm{~K}^{-1}$ for $\mathrm{HfSe}_{2}$, which is lower than those values of $\mathrm{MoS}_{2}$-type monolayers ${ }^{[79,92-93]}$ and comparable to those of some popular bulk TE materials, such as $\mathrm{Bi}_{2} \mathrm{Te}_{3}{ }^{[94]}$ and $\mathrm{PbTe}^{[95]}$.

Recently, another class highly desirable 2D material has been theoretically predicated and explored experimentally ${ }^{[96-100]}$. They are formed by the combination of chalcogens and noble metals, such as Pt and Pd. They have usually bulked or puckered pentagonal structure with low symmetry. Oyedele et al. has been exfoliated monolayer PdSe $e_{2}$ from its bulk crystals ${ }^{[98]}$. This typical 2D noble transition metal dichalcogenide monolayer with a puckered morphology is air-stable. The theoretical results demonstrate that the mobility of holes can reach 1929 $\mathrm{cm}^{2} \mathrm{~V}^{-1} \mathrm{~s}^{-1}$ for monolayer $\mathrm{PdSe}_{2}$. It possesses the lower lattice thermal conductivity of $3.7 \mathrm{Wm}^{-1} \mathrm{~K}^{-1[100]}$, which is much lower than that of $\mathrm{MoS}_{2}$. It indicates that $\mathrm{PdSe}_{2}$ monolayer could have a quite potential for TE applications. Recently, a novel $\mathrm{Pd}_{2} \mathrm{Se}_{3}$ monolayer phase, which is reconstructed from a few layers of $\mathrm{PdSe}_{2}$ by the interlayer fusion, has been reported experimentally ${ }^{[101]}$. Figure $4 d$ shows the experimental TEM image with atomic resolution (grey) of the monolayer and correspondingly theoretical TEM image by the simulation. The ultralow lattice thermal conductivity has been found in $\mathrm{Pd}_{2} \mathrm{Se}_{3}$ monolayer. Theoretical analysis reveals that in the region of low frequency a strong hybridization is between $\left[\mathrm{Se}_{2}\right]^{2-}$ ion and $\mathrm{Pd}^{2+}$, as verified by the phonon DOS in figure $4 \mathrm{e}^{[102]}$. Thus, it is proposed that the low thermal conductivity is due to the softening of phonon modes and the anharmonicity between covalently bonded $\left[\mathrm{Se}_{2}\right]^{2-}$. The lattice thermal conductivity of $\mathrm{Pd}_{2} \mathrm{Se}_{3}$ is calculated and is about $2 \mathrm{Wm}^{-1} \mathrm{~K}^{-1}$. This small value is 90 , 27 , and 6 times lower than that of $\mathrm{MoS}_{2}^{[103]}$, WSe ${ }_{2}^{[79]}$, and $\mathrm{TiS}_{3}{ }^{[104]}$, respectively.

\section{Potential applications of two-dimensional thermoelectric materials}

Traditionally, TE materials are mainly used as TE power generation and TE refrigeration. The TE generator can be applied in the TE power station with the long distance and unmanned maintenance, such as in man-made satellite. They can be applied in the low-grade TE generation, such as industrial waste heat, waste heat in our daily life and heats from vehicle exhaust. TE materials with the characteristics of both cooling and heating can control easily the temperature timing and are used to provide the constant temperature environment for different systems, such as electronic computers, cameras with CCD and communication devices. They are also used as the sensors and temperature controllers with extensive applications in microelectronic systems and devices. Here, we won't discuss these traditional applications in details. Following the device miniaturization, TE materials will expand their applications in other potential areas further. Especially, two-dimensional TE materials have wide application prospect in wearable/flexible systems.

With the recent appearing of the IoT era, and the wide range use of microelectronic devices by that people can communicate with each other at anytime and anywhere, the research of wearable electronic systems is growing. However, it still remains a problem for the power supplies of wearables. At present, batteries can power these wearable devices. But the frequent recharging and replacement are unavoidable problems. To solve this issue, one of most effective strategy is to integrate self-powered system with wearable electronic devices. Here, the energy conversion by TE effect on basis of large-area flexible materials is expected to serve as the energy harvester with selfpowered way. This has received large attention because of its advantages ${ }^{[105-109]}$. For examples, the heat energy from body may be enough and the long lifetime prevents them from frequent replacement. In addition, the TE conversion process don't create any noise ${ }^{14}$. 
(a)
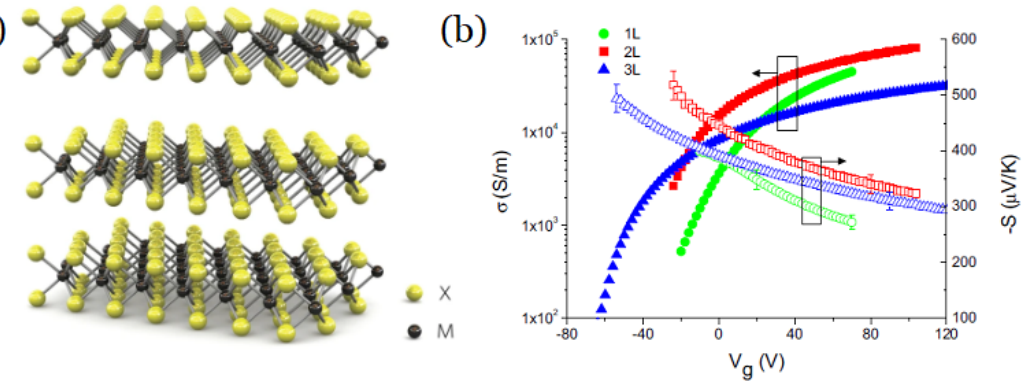

(c)

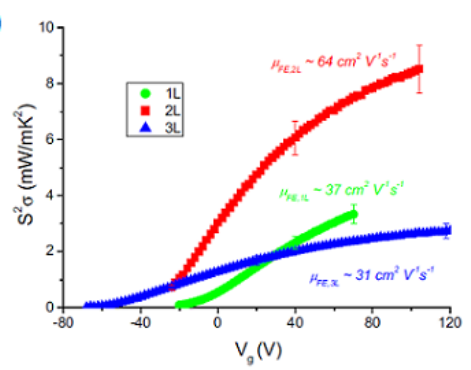

(d)
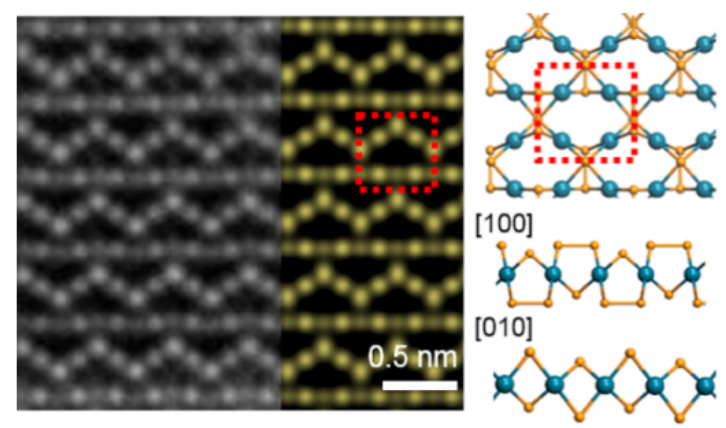

(e)

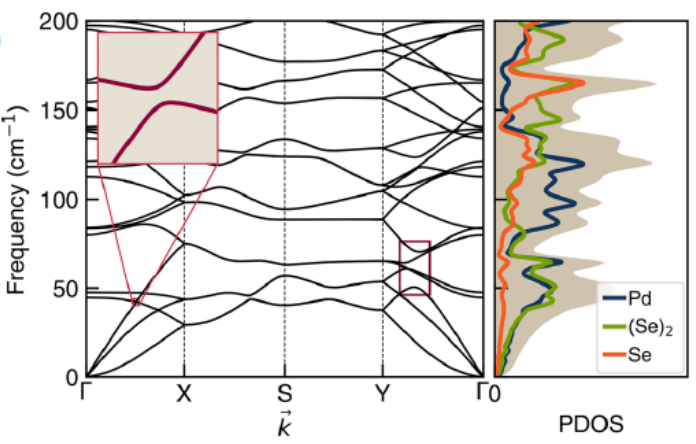

Figure 4 (a) Three-dimensional schematic representation of typical hexagonal $\mathrm{MX}_{2}$ structure. Reproduced with permission from ref. 77. Copyright 2012, NPG. (b) Electrical conductivities $\sigma$ and Seebeck coefficients $S$ at RT as functions of $V_{\text {g }}$ for single layer $\mathrm{MoS}_{2}$. (c) Power factor $S^{2} \sigma$ as a function of $V_{\mathrm{g}}$. Reproduced with permission from ref. 87. Copyright 2017, APS. (d) High resolution TEM (grey) and simulated (yellow) image for monolayer $\mathrm{Pd}_{2} \mathrm{Se}_{3}$. Reproduced with permission from ref. 101. Copyright 2017, APS. (e) Phonon band structure and density of states of monolayer $\mathrm{Pd}_{2} \mathrm{Se}_{3}$. Reproduced with permission from ref. 102. Copyright 2018, ACS.

(a)

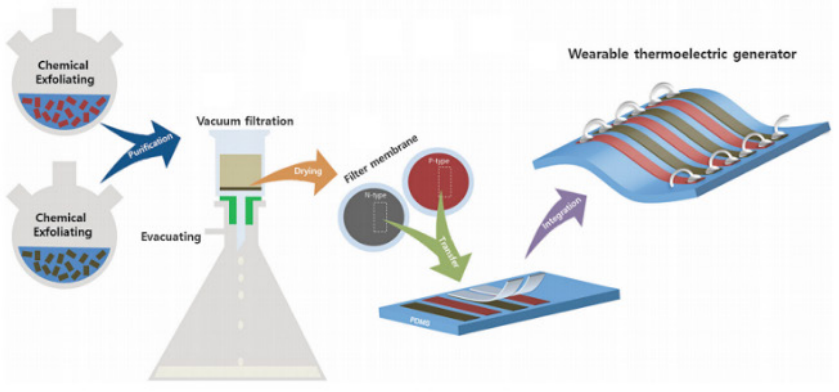

(c)

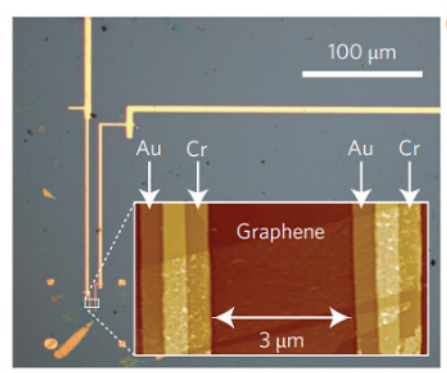

(d)

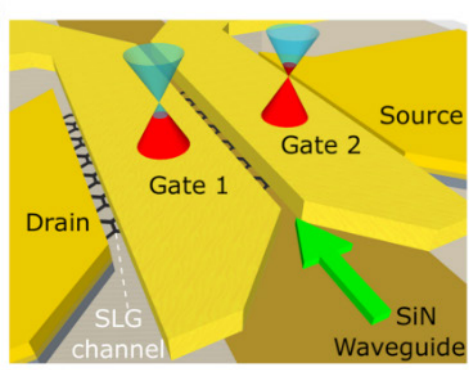

(b)
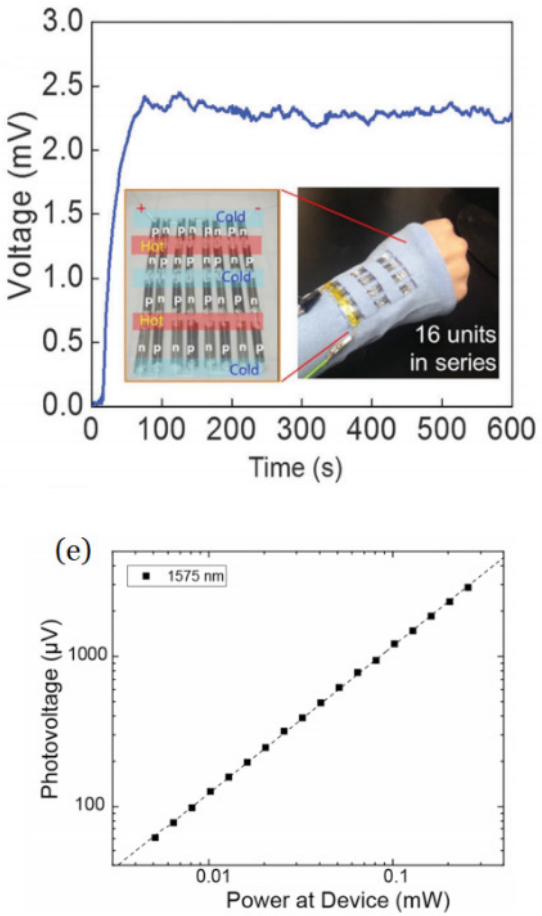

Figure 5 (a) Fabrication processes of wearable thermoelectric devices using TMDs nanosheets. (b) Output voltage from the human wrist's heat by thermoelectric generator. Reproduced with permission from Ref. 111. Copyright 2016, RSC. (c) Optical micrograph and atomic force microscopy of graphene photo detector. Reproduced with permission from Ref. 112. Copyright 2014, NPG. (d) Schematic illustration of the graphene photo detector with SiN waveguide. (e) The relation between photo voltage and optical power. Reproduced with permission from ref. 113. Copyright 2019, ACS. 
Originally, the TE generator consisting of bulk $\mathrm{Bi}_{2} \mathrm{Te}_{3}$ and $\mathrm{Sb}_{2} \mathrm{Te}_{3}$ printed on a flexible glass fabric has been reported $^{[107]}$. A bend ability radius of up to $20 \mathrm{~mm}$ is shown in this kind of devices. This basically meets the demand of flexible wearables. Kim et al. ${ }^{[10]}$ have fabricated a novel wearable TE generator, which could attach to human skin and use the heat energy from body. Thus, it is expected to be with various potential applications. Because of unique electrical and mechanical properties, TMDs have given risen to great attention due to their potential in nextgeneration microelectronics and wearables. Oh et al. ${ }^{[111]}$ developed a method by combining chemical exfoliation and vacuum filtration to construct the TE generators directly which are parallel-connected on flexible substrates (seen in figure 5a). As shown in figure 5b, $\mathrm{NbSe}_{2}$ and $\mathrm{WS}_{2}$ nanosheets were used as the $p$-type and $n$-type materials, respectively. In this way, the TE generators were fabricated. This kind of devices has very high flexibility and durability. They can be used as the glove-type wrist-band thermal sensor.

Moreover, based on the Seebeck effect, the photo TE device can generate a photocurrent. With the adsorbed light, the temperature gradient between lighting region and dark region is built and thus the electric voltage is formed. This phenomenon has several advantages in designing the optoelectronics devices with high performance. The graphene TE terahertz photo detector has been investigated ${ }^{[12]}$ (figure $5 \mathrm{c}$ ). This kind of device demonstrates an outstanding sensitivity more than 10 $\mathrm{VW}^{-1}$ at RT with noise-equivalent power less than 1100 $\mathrm{pW} \mathrm{Hz}{ }^{-1 / 2}$, referenced to the incident power. If based on absorbed power, the sensitivity exceeds $700 \mathrm{VW}^{-1}$ at RT with noise-equivalent power less than $20 \mathrm{pW} \mathrm{Hz}^{-1 / 2}$. A photo-thermoelectric-based graphene photo detector has been designed to generate a photovoltage directly (seen in figure $5 \mathrm{~d})^{[113]}$. It exhibits an external responsivity of about $12.2 \mathrm{~V} / \mathrm{W}$ with a roll-off frequency up to $42 \mathrm{GHz}$ and $3 \mathrm{~dB}$ bandwidth. Figure $5 \mathrm{e}$ shows the relation between photovoltage and optical power. With TE effect, the voltage generated by temperature gradient due to the lighting has a linear response to optical power ${ }^{[113]}$.

\section{Conclusion and Outlook}

Over the last few decades, TE materials have made a great progress. In this review, we are focused on the TE properties of new two-dimensional materials and the possible applications. In particular, the relevant theoretical and experimental results for group IV-VI, group IV-V, group III-VI and layered transition metal dichalcogenides have been highlighted. We also compared the TE properties between new two-dimensional materials we considered and state-of-the-art layered structure materials. In these new two-dimensional materials, both low thermal conductivity and high power factor have been revealed. Finally, the current potential applications in TE device, including flexible generator and optoelectronics devices using twodimensional film materials have also been presented.

Although the tremendous advances have been achieved during the past decades, there are still problems and challenges for the practical applications. The phonon and electrical transport mechanism are still obscure. The mechanism of increasing the power factor has been proposed in two-dimension materials, but it still remains challenge to decouple the different transport coefficients. We also need a deeper understanding of transport mechanisms to help enhance TE properties and design new TE materials in the future.

Conflicts of interest: The authors declare no competing financial interest.

Acknowledgments: Thanks for the support of the National Key R\&D Program of China under Grant No. 2016YFA0200400 and the National Natural Science Foundation of China under Grant No. 51627805.

\section{References}

[1] Bell, L. E., Cooling, Heating, Generating Power, and Recovering Waste Heat with Thermoelectric Systems. Science 2008,321 (5895), 1457-1461.

[2] Pichanusakorn, P.; Bandaru, P., Nanostructured thermoelectrics. Mater. Sci. Eng., R 2010,67, 19-63.

[3] Rull-Bravo, M.; Moure, A.; Fernandez, J.; Martín-González, M., Skutterudites as thermoelectric materials: revisited. RSC Adv. 2015,5, 41653-41667.

[4] Sundarraj, P.; Maity, D.; Roy, S. S.; Taylor, R. A., Recent advances in thermoelectric materials and solar thermoelectric generators-a critical review. RSC Adv. 2014,4, 46860-46874.

[5] Tan, G.; Zhao, L.-D.; Kanatzidis, M. G., Rationally designing high-performance bulk thermoelectric materials. Chem. Rev. 2016,116, 12123-12149.

[6] Snyder, G. J.; Toberer, E. S., Complex thermoelectric materials. Nat. Mater. 2008,7, 105-114.

[7] Heremans, J. P.; Jovovic, V.; Toberer, E. S.; Saramat, A.; Kurosaki, K.; Charoenphakdee, A.; Yamanaka, S.; Snyder, G. J., Enhancement of thermoelectric efficiency in PbTe by distortion of the electronic density of states. Science 2008,321, 554-557.

[8] Pei, Y.; LaLonde, A. D.; Heinz, N. A.; Shi, X.; Iwanaga, S.; Wang, H.; Chen, L.; Snyder, G. J., Stabilizing the optimal carrier concentration for high thermoelectric efficiency. Adv. Mater. 2011,23, 5674-5678.

[9] Pei, Y.; Shi, X.; LaLonde, A.; Wang, H.; Chen, L.; Snyder, G. J., Convergence of electronic bands for high performance bulk thermoelectrics. Nature 2011,473, 66.

[10] Liu, W.; Tan, X.; Yin, K.; Liu, H.; Tang, X.; Shi, J.; Zhang, Q.; Uher, C., Convergence of conduction bands as a means of enhancing thermoelectric performance of $\mathrm{n}$-type $\mathrm{Mg}$ 2 Si 1- x Sn x solid solutions. Phys. Rev. Lett. 2012,108, 166601.

[11] Heremans, J. P.; Wiendlocha, B.; Chamoire, A. M., Resonant levels in bulk thermoelectric semiconductors. Energy Environ. Sci. 2012,5, 5510-5530. 
[12] Tan, G.; Shi, F.; Hao, S.; Chi, H.; Zhao, L.-D.; Uher, C.; Wolverton, C.; Dravid, V. P.; Kanatzidis, M. G., Codoping in SnTe: Enhancement of thermoelectric performance through synergy of resonance levels and band convergence. J. Am. Chem. Soc. 2015,137, 5100-5112.

[13] C.-W. Yau; T. T.-O. Kwok; C.-U. Lei; Kwok, Y.-K., in Internet of everything: Algorithms, methodologies, technologies and perspectives. Springer Singapore: (Eds:B. Di Martino, K.-C. Li, L. T. Yang, A. Esposito), SpringerSingapore, Singapore, 2018.

[14] Kanahashi, K.; Pu, J.; Takenobu, T., 2D Materials for Large-Area Flexible Thermoelectric Devices. Adv. Energy Mater. 2020,10, 1902842.

[15] Li, D.; Gong, Y.; Chen, Y.; Lin, J.; Khan, Q.; Zhang, Y.; Li, Y.; Zhang, H.; Xie, H., Recent Progress of Two-Dimensional Thermoelectric Materials. Nano-Micro Lett. 2020,12, 36.

[16] Dresselhaus, M. S.; Chen, G.; Tang, M. Y.; Yang, R.; Lee, H.; Wang, D.; Ren, Z.; Fleurial, J. P.; Gogna, P., New directions for low-dimensional thermoelectric materials. Adv. Mater. 2007,19 (8), 1043-1053.

[17] Hicks, L.; Dresselhaus, M. S., Effect of quantum-well structures on the thermoelectric figure of merit. Phys. Rev. B 1993,47, 12727.

[18] Heremans, J. P., Low-Dimensional Thermoelectricity. Acta Phys. Polon. A 2005,108, 609-634.

[19] Schedin, F.; Geim, A. K.; Morozov, S. V.; Hill, E. W.; Blake, P.; Katsnelson, M. I.; Novoselov, K. S., Detection of individual gas molecules adsorbed on graphene. Nat. Mater. 2007,6, 652-655.

[20] Chang, C.; Wu, M.; He, D.; Pei, Y.; Wu, C.-F.; Wu, X.; Yu, H.; Zhu, F.; Wang, K.; Chen, Y.; Li, H.; Jing-Feng, L.; Jiaqing, H.; Li-Dong, Z., 3D charge and 2D phonon transports leading to high out-of-plane ZT in n-type SnSe crystals. Science 2018,360, 778-783.

[21] Zhao, L.-D.; Lo, S.-H.; Zhang, Y.; Sun, H.; Tan, G.; Uher, C.; Wolverton, C.; Dravid, V. P.; Kanatzidis, M. G., Ultralow thermal conductivity and high thermoelectric figure of merit in SnSe crystals. Nature 2014,508, 373.

[22] Liu, Y.; Zhang, S.; He, J.; Wang, Z. M.; Liu, Z., Recent Progress in the Fabrication, Properties, and Devices of Heterostructures Based on 2D Materials. Nano-Micro Lett. 2019,11, 13.

[23] Molle, A.; Goldberger, J.; Houssa, M.; Xu, Y.; Zhang, S.-C.; Akinwande, D., Buckled two-dimensional Xene sheets. Nat. Mater. 2017,16, 163-169.

[24] Li, L.; Yu, Y.; Ye, G. J.; Ge, Q.; Ou, X.; Wu, H.; Feng, D.; Chen, X. H.; Zhang, Y., Black phosphorus field-effect transistors. Nat. Nanotechnol. 2014,9, 372-377.

[25] Lee, C.; Wei, X.; Kysar, J. W.; Hone, J., Measurement of the Elastic Properties and Intrinsic Strength of Monolayer Graphene. Science 2008,321, 385-388.

[26] Yang, J.; Lü, T.; Myint, Y. W.; Pei, J.; Macdonald, D.; Zheng, J.-C.; Lu, Y., Robust Excitons and Trions in Monolayer
MoTe2. ACS Nano 2015,9, 6603-6609.

[27] Lai, J.; Liu, X.; Ma, J.; Wang, Q.; Zhang, K.; Ren, X.; Liu, Y.; Gu, Q.; Zhuo, X.; Lu, W.; Wu, Y.; Li, Y.; Feng, J.; Zhou, S.; Chen, J.-H.; Sun, D., Anisotropic Broadband Photoresponse of Layered Type-II Weyl Semimetal MoTe2. Adv. Mater. 2018,30, 1707152.

[28] Nie, C.; Yu, L.; Wei, X.; Shen, J.; Lu, W.; Chen, W.; Feng, S.; Shi, H., Ultrafast growth of large-area monolayer MoS2film via gold foil assistant CVD for a highly sensitive photodetector. Nanotechnol. 2017,28, 275203.

[29] Venkatasubramanian, R.; Siivola, E.; Colpitts, T.; O'quinn, B., Thin-film thermoelectric devices with high roomtemperature figures of merit. Nature 2001,413, 597.

[30] Wu, C.-W.; Wu, Y.-R., Optimization of thermoelectric properties for rough nano-ridge GaAs/AlAs superlattice structure. AIP Adv. 2016,6, 115201.

[31] Ohta, H.; Kim, S.; Mune, Y.; Mizoguchi, T.; Nomura, K.; Ohta, S.; Nomura, T.; Nakanishi, Y.; Ikuhara, Y.; Hirano, M.; Hideo, H.; Kunihito, K., Giant thermoelectric Seebeck coefficient of a two-dimensional electron gas in SrTiO 3. Nat. Mater. 2007,6, 129.

[32] Liu, Y.; Wang, W.; Yang, J.; Li, S., Recent Advances of Layered Thermoelectric Materials. Adv. Sustainable Syst. 2018,2, 1800046.

[33] Singh, A. K.; Mathew, K.; Zhuang, H. L.; Hennig, R. G., Computational Screening of 2D Materials for Photocatalysis. J. Phys. Chem. Lett. 2015,6, 1087-1098.

[34] Singh, A. K.; Hennig, R. G., Computational prediction of two-dimensional group-IV mono-chalcogenides. Appl. Phys. Lett. 2014,105, 042103.

[35] Wang, F. Q.; Zhang, S.; Yu, J.; Wang, Q., Thermoelectric properties of single-layered SnSe sheet. Nanoscale 2015,7, 15962-15970.

[36] Volykhov, A.; Shtanov, V.; Yashina, L., Phase relations between germanium, tin, and lead chalcogenides in pseudobinary systems containing orthorhombic phases. Inorg. Mater. 2008,44, 345-356.

[37] Hu, Z.-Y.; Li, K.-Y.; Lu, Y.; Huang, Y.; Shao, X.-H., High thermoelectric performances of monolayer SnSe allotropes. Nanoscale 2017,9, 16093-16100.

[38] Morales-Ferreiro, J.; Diaz-Droguett, D.; Celentano, D.; Luo, T., First-Principles Calculations of Thermoelectric Properties of IV-VI Chalcogenides 2D Materials. Front. Mech. Eng. 2017,3, 15.

[39] Guo, S.-D.; Wang, Y.-H., Thermoelectric properties of orthorhombic group IV-VI monolayers from the firstprinciples calculations. J. Appl. Phys. 2017,121, 034302.

[40] Xu, L.; Yang, M.; Wang, S. J.; Feng, Y. P., Electronic and optical properties of the monolayer group-IV monochalcogenides $\mathrm{MX}(\mathrm{M}=\mathrm{Ge}, \mathrm{Sn} ; \mathrm{X}=\mathrm{S}, \mathrm{Se}, \mathrm{Te})$. Phys. Rev. B 2017,95, 235434.

[41] Shafique, A.; Shin, Y.-H., Thermoelectric and phonon transport properties of two-dimensional IV-VI 
compounds. Sci. Rep. 2017,7, 506.

[42] Qin, G.; Qin, Z.; Fang, W.-Z.; Zhang, L.-C.; Yue, S.-Y.; Yan, Q.-B.; Hu, M.; Su, G., Diverse anisotropy of phonon transport in two-dimensional group IV-VI compounds: A comparative study. Nanoscale 2016,8, 11306-11319.

[43] Ding, G.; Gao, G.; Yao, K., High-efficient thermoelectric materials: The case of orthorhombic IV-VI compounds. Sci. Rep. 2015,5, 9567.

[44] Yang, J.-H.; Yuan, Q.; Deng, H.; Wei, S.-H.; Yakobson, B. I., Earth-abundant and non-toxic SiX (X=S, Se) monolayers as highly efficient thermoelectric materials. J. Phys. Chem. C 2017,121, 123-128.

[45] Nissimagoudar, A. S.; Ma, J.; Chen, Y.; Li, W., Thermal transport in monolayer InSe. J. Phys. Condens. Matter 2017,29, 335702.

[46] Bahuguna, B. P.; Saini, L.; Sharma, R. O.; Tiwari, B., Hybrid functional calculations of electronic and thermoelectric properties of GaS, GaSe, and GaTe monolayers. Phys. Chem. Chem. Phys. 2018,20, 28575-28582.

[47] Huang, H.; Xing, G.; Fan, X.; Singh, D. J.; Zheng, W., Layered TI 2 O: a model thermoelectric material. J. Mater. Chem. C 2019,7, 5094-5103.

[48] Han, G.; Chen, Z. G.; Drennan, J.; Zou, J., Indium selenides: structural characteristics, synthesis and their thermoelectric performances. Small 2014,10, 27472765.

[49] Hogg, J.; Sutherland, H.; Williams, D., The crystal structure of tetraindium triselenide. Acta Crystallogr., Sect. B: Struct. Crystallogr. Cryst. Chem. 1973,29, 15901593.

[50] Popović, S.; Tonejc, A.; Gržeta-Plenković, B.; Čelustka, B.; Trojko, R., Revised and new crystal data for indium selenides. J. Appl. Crystallogr. 1979,12 (4), 416-420.

[51] Nagpal, K.; Ali, S., X-Ray Crystallographic Study of InSe. Indian J. Pure Appl. Phys. 1976,14, 434-440.

[52] Hogg, J., The crystal structure of In6Se7. Acta Crystallogr. Sect. B Struct. Crystallogr. Cryst. Chem. 1971,27, 16301634.

[53] Han, G.; Chen, Z.-G.; Yang, L.; Cheng, L.; Jack, K.; Drennan, J.; Zou, J., Thermal stability and oxidation of layer-structured rhombohedral In3Se4 nanostructures. Appl. Phys. Lett. 2013,103, 263105.

[54] Bandurin, D. A.; Tyurnina, A. V.; Geliang, L. Y.; Mishchenko, A.; Zólyomi, V.; Morozov, S. V.; Kumar, R. K.; Gorbachev, R. V.; Kudrynskyi, Z. R.; Pezzini, S.; D., K. Z.; Uli, Z.; S., N. K.; Amalia, P.; Laurence, E.; V., G. I.; I., F. K. V.; K., G. A.; Yang, C., High electron mobility, quantum Hall effect and anomalous optical response in atomically thin InSe. Nat. Nanotechnol. 2017,12, 223.

[55] Rhyee, J.-S.; Lee, K. H.; Lee, S. M.; Cho, E.; Kim, S. I.; Lee, E.; Kwon, Y. S.; Shim, J. H.; Kotliar, G., Peierls distortion as a route to high thermoelectric performance in $\ln _{4} \mathrm{Se}_{3-\delta}$ crystals. Nature 2009,459, 965.
[56] Zolyomi, V.; Drummond, N.; Fal'Ko, V., Band structure and optical transitions in atomic layers of hexagonal gallium chalcogenides. Phys. Rev. B 2013,87, 195403.

[57] Zólyomi, V.; Drummond, N.; Fal'Ko, V., Electrons and phonons in single layers of hexagonal indium chalcogenides from ab initio calculations. Phys. Rev. B 2014,89, 205416.

[58] Hu, P.; Wang, L.; Yoon, M.; Zhang, J.; Feng, W.; Wang, X.; Wen, Z.; Idrobo, J. C.; Miyamoto, Y.; Geohegan, D. B.; Kai, X., Highly responsive ultrathin GaS nanosheet photodetectors on rigid and flexible substrates. Nano Lett. 2013,13, 1649-1654.

[59] Das, P.; Wickramaratne, D.; Debnath, B.; Yin, G.; Lake, R. K., Charged impurity scattering in two-dimensional materials with ring-shaped valence bands: GaS, GaSe, InS, and InSe. Phys. Rev. B 2019,99, 085409.

[60] Lei, S.; Ge, L.; Liu, Z.; Najmaei, S.; Shi, G.; You, G.; Lou, J.; Vajtai, R.; Ajayan, P. M., Synthesis and photoresponse of large GaSe atomic layers. Nano Lett. 2013,13, 2777-2781.

[61] Lei, S.; Ge, L.; Najmaei, S.; George, A.; Kappera, R.; Lou, J.; Chhowalla, M.; Yamaguchi, H.; Gupta, G.; Vajtai, R.; D., M. A.; M., A. P., Evolution of the electronic band structure and efficient photo-detection in atomic layers of InSe. ACS nano 2014,8, 1263-1272.

[62] Hu, P.; Wen, Z.; Wang, L.; Tan, P.; Xiao, K., Synthesis of few-layer GaSe nanosheets for high performance photodetectors. ACS nano 2012,6, 5988-5994.

[63] Late, D. J.; Liu, B.; Luo, J.; Yan, A.; Matte, H. R.; Grayson, M.; Rao, C.; Dravid, V. P., GaS and GaSe ultrathin layer transistors. Adv. Mater. 2012,24, 3549-3554.

[64] Wickramaratne, D.; Zahid, F.; Lake, R. K., Electronic and thermoelectric properties of van der Waals materials with ring-shaped valence bands. J. Appl. Phys. 2015,118, 075101.

[65] Hung, N. T.; Nugraha, A. R.; Saito, R., Two-dimensional InSe as a potential thermoelectric material. Appl. Phys. Lett. 2017,111, 092107.

[66] T. Hung, N.; Nugraha, A. R.; Yang, T.; Zhang, Z.; Saito, R., Thermoelectric performance of monolayer InSe improved by convergence of multivalley bands. J. Appl. Phys. 2019,125, 082502.

[67] Zeng, J.; He, X.; Liang, S.-J.; Liu, E.; Sun, Y.; Pan, C.; Wang, Y.; Cao, T.; Liu, X.; Wang, C.; Lili, Z.; Shengnan, Y.; Guangxu, S.; Zhenlin, W.; Kenji, W.; Takashi, T.; J., S. D.; Lijun, Z.; Feng, M., Experimental identification of critical condition for drastically enhancing thermoelectric power factor of two-dimensional layered materials. Nano Lett. 2018,18, 7538-7545.

[68] Balendhran, S.; Walia, S.; Nili, H.; Sriram, S.; Bhaskaran, M., Elemental analogues of graphene: silicene, germanene, stanene, and phosphorene. Small 2015,11, 640-652.

[69] Zhang, S.; Yan, Z.; Li, Y.; Chen, Z.; Zeng, H., Atomically thin 
arsenene and antimonene: semimetal-semiconductor and indirect-direct band-gap transitions. Angew. Chem. Int. Ed. 2015,54, 3112-3115.

[70] Huang, H.; Fan, X.; Singh, D. J.; Zheng, W., The thermal and thermoelectric transport properties of SiSb, GeSb and SnSb monolayers. J. Mater. Chem. C 2019,7, 1065210662.

[71] Barreteau, C.; Michon, B.; Besnard, C.; Giannini, E., Highpressure melt growth and transport properties of SiP, SiAs, GeP, and GeAs 2D layered semiconductors. J. Cryst. Growth 2016,443, 75-80.

[72] Lee, K.; Kamali, S.; Ericsson, T.; Bellard, M.; Kovnir, K., GeAs: Highly anisotropic van der Waals thermoelectric material. Chem. Mater. 2016,28, 2776-2785.

[73] Wang, F. Q.; Guo, Y.; Wang, Q.; Kawazoe, Y.; Jena, P., Exceptional thermoelectric properties of layered GeAs2. Chem. Mater. 2017,29, 9300-9307.

[74] Zhao, L.-D.; Tan, G.; Hao, S.; He, J.; Pei, Y.; Chi, H.; Wang, H.; Gong, S.; Xu, H.; Dravid, V. P.; Ctirad, U.; Jeffrey, S. G.; Chris, W.; G., K. M., Ultrahigh power factor and thermoelectric performance in hole-doped single-crystal SnSe. Science 2016,351, 141-144.

[75] Zhao, T.; Sun, Y.; Shuai, Z.; Wang, D., GeAs2: A IV-V group two-dimensional semiconductor with ultralow thermal conductivity and high thermoelectric efficiency. Chem. Mater. 2017,29, 6261-6268.

[76] Zhang, W.; Huang, Z.; Zhang, W.; Li, Y., Two-dimensional semiconductors with possible high room temperature mobility. Nano Res. 2014,7, 1731-1737.

[77] Wang, Q. H.; Kalantar-Zadeh, K.; Kis, A.; Coleman, J. N.; Strano, M. S., Electronics and optoelectronics of twodimensional transition metal dichalcogenides. Nat. Nanotechnol. 2012,7, 699-712.

[78] Ghosh, K.; Singisetti, U., Thermoelectric transport coefficients in mono-layer MoS2 and WSe2: role of substrate, interface phonons, plasmon, and dynamic screening. J. Appl. Phys. 2015,118, 135711.

[79] Kumar, S.; Schwingenschlogl, U., Thermoelectric response of bulk and monolayer MoSe2 and WSe2. Chem. Mater. 2015,27, 1278-1284.

[80] Li, W.; Carrete, J.; Mingo, N., Thermal conductivity and phonon linewidths of monolayer MoS2 from first principles. Appl. Phys. Lett. 2013,103, 253103.

[81] Zhang, G.; Zhang, Y.-W., Thermoelectric properties of two-dimensional transition metal dichalcogenides. J. Mater. Chem. C 2017,5, 7684-7698.

[82] Yan, R.; Simpson, J. R.; Bertolazzi, S.; Brivio, J.; Watson, M.; Wu, X.; Kis, A.; Luo, T.; Hight Walker, A. R.; Xing, H. G., Thermal conductivity of monolayer molybdenum disulfide obtained from temperature-dependent Raman spectroscopy. ACS nano 2014,8, 986-993.

[83] Mak, K. F.; Lee, C.; Hone, J.; Shan, J.; Heinz, T. F., Atomically thin $\mathrm{MoS}_{2}$ : a new direct-gap semiconductor. Phys. Rev.
Lett. 2010,105, 136805

[84] Fan, X.; Chang, C.-H.; Zheng, W.; Kuo, J.-L.; Singh, D. J., The electronic properties of single-layer and multilayer $\mathrm{MoS}_{2}$ under high pressure. J. Phys. Chem. C 2015,119, 10189-10196.

[85] Dou, X.; Ding, K.; Jiang, D.; Sun, B., Tuning and Identification of Interband Transitions in Monolayer and Bilayer Molybdenum Disulfide Using Hydrostatic Pressure. ACS Nano 2014,8, 7458-7464.

[86] Fan, X.; Singh, D. J.; Zheng, W., Valence band splitting on multilayer Mos2: mixing of spin-orbit coupling and interlayer coupling. J. Phys. Chem. Lett. 2016,7, 21752181.

[87] Hippalgaonkar, K.; Wang, Y.; Ye, Y.; Qiu, D. Y.; Zhu, H.; Wang, Y.; Moore, J.; Louie, S. G.; Zhang, X., High thermoelectric power factor in two-dimensional crystals of $\mathrm{MoS}_{2}$. Phys. Rev. B 2017,95, 115407.

[88] Wickramaratne, D.; Zahid, F.; Lake, R. K., Electronic and thermoelectric properties of few-layer transition metal dichalcogenides. J. Chem. Phys. 2014,140, 124710.

[89] Huang, W.; Luo, X.; Gan, C. K.; Quek, S. Y.; Liang, G., Theoretical study of thermoelectric properties of fewlayer $\mathrm{MoS}_{2}$ and $\mathrm{WSe}_{2}$. Phys. Chem. Chem. Phys. 2014,16, 10866-10874.

[90] Yumnam, G.; Pandey, T.; Singh, A. K., High temperature thermoelectric properties of $\mathrm{Zr}$ and $\mathrm{Hf}$ based transition metal dichalcogenides: A first principles study. J. Chem. Phys. 2015,143, 234704.

[91] Ding, G.; Gao, G.; Huang, Z.; Zhang, W.; Yao, K., Thermoelectric properties of monolayer MSe2 ( $M=Z r$, $\mathrm{Hf}$ ): low lattice thermal conductivity and a promising figure of merit. Nanotechnol. 2016,27, 375703.

[92] Jin, Z.; Liao, Q.; Fang, H.; Liu, Z.; Liu, W.; Ding, Z.; Luo, T.; Yang, N., A revisit to high thermoelectric performance of single-layer MoS 2. Sci. Rep. 2015,5, 18342.

[93] Sahoo, S.; Gaur, A. P.; Ahmadi, M.; Guinel, M. J.-F.; Katiyar, R. S., Temperature-dependent Raman studies and thermal conductivity of few-layer MoS2. J. Phys. Chem. C 2013,117, 9042-9047.

[94] Hellman, O.; Broido, D. A., Phonon thermal transport in $\mathrm{Bi}_{2} \mathrm{Te}_{3}$ from first principles. Phys. Rev. B 2014,90, 134309.

[95] Qiu, B.; Bao, H.; Ruan, X.; Zhang, G.; Wu, Y. In Molecular dynamics simulations of lattice thermal conductivity and spectral phonon mean free path of PbTe: Bulk and nanostructures, Comput. Mater. Sci., 2012; pp 278-285.

[96] Sun, J.; Shi, H.; Siegrist, T.; Singh, D. J., Electronic, transport, and optical properties of bulk and mono-layer PdSe2. Appl. Phys. Lett. 2015,107, 153902.

[97] Chow, W. L.; Yu, P.; Liu, F.; Hong, J.; Wang, X.; Zeng, Q.; Hsu, C. H.; Zhu, C.; Zhou, J.; Wang, X.; Juan, X.; Jiaxu, Y.; Yu, C.; Di, W.; Ting, Y.; Zexiang, S.; Hsin, L.; Chuanhong, J.; Kang, T. B.; Zheng, L., High mobility 2D palladium diselenide field-effect transistors with tunable ambipolar 
characteristics. Adv. Mater. 2017,29, 1602969.

[98] Oyedele, A. D.; Yang, S.; Liang, L.; Puretzky, A. A.; Wang, K.; Zhang, J.; Yu, P.; Pudasaini, P. R.; Ghosh, A. W.; Liu, Z., PdSe2: Pentagonal two-dimensional layers with high air stability for electronics. J. Am. Chem. Soc. 2017,139, 14090-14097.

[99] Wang, Y.; Li, Y.; Chen, Z., Not your familiar two dimensional transition metal disulfide: structural and electronic properties of the PdS 2 monolayer. J. Mater. Chem. C 2015,3, 9603-9608.

[100] Qin, D.; Yan, P.; Ding, G.; Ge, X.; Song, H.; Gao, G., Monolayer PdSe2: A promising two-dimensional thermoelectric material. Sci. Rep. 2018,8, 2764.

[101] Lin, J.; Zuluaga, S.; Yu, P.; Liu, Z.; Pantelides, S. T.; Suenaga, K., Novel Pd2Se3 Two-Dimensional Phase Driven by Interlayer Fusion in Layered PdSe 2. Phys. Rev. Lett. 2017,119, 016101.

[102] Naghavi, S. S.; He, J.; Xia, Y.; Wolverton, C., Pd2Se3 monolayer: a promising two-dimensional thermoelectric material with ultralow lattice thermal conductivity and high power factor. Chem. Mater. 2018,30, 5639-5647.

[103] Gandi, A. N.; Schwingenschlögl, U., Thermal conductivity of bulk and monolayer MoS2. EPL 2016,113, 36002.

[104] Zhang, J.; Liu, X.; Wen, Y.; Shi, L.; Chen, R.; Liu, H.; Shan, B., Titanium trisulfide monolayer as a potential thermoelectric material: a first-principles-based Boltzmann transport study. ACS Appl. Mater. Interfaces 2017,9, 2509-2515.

[105] Park, S. H.; Jo, S.; Kwon, B.; Kim, F.; Ban, H. W.; Lee, J. E.; Gu, D. H.; Lee, S. H.; Hwang, Y.; Kim, J.-S.; DowBin, H.; Sukbin, L.; Jin, C. K.; Wook, J.; Sung, S. J., Highperformance shape-engineerable thermoelectric painting. Nat. Commun. 2016,7, 1-10.

[106] Suemori, K.; Watanabe, Y.; Hoshino, S., Carbon nanotube bundles/polystyrene composites as high- performance flexible thermoelectric materials. Appl. Phys. Lett. 2015,106, 113902.

[107] Kim, S. J.; We, J. H.; Cho, B. J., A wearable thermoelectric generator fabricated on a glass fabric. Energy Environ. Sci. 2014,7, 1959-1965.

[108] Lu, Z.; Layani, M.; Zhao, X.; Tan, L. P.; Sun, T.; Fan, S.; Yan, Q.; Magdassi, S.; Hng, H. H., Fabrication of flexible thermoelectric thin film devices by inkjet printing. Small 2014,10, 3551.

[109] Kim, S. J.; Choi, H.; Kim, Y.; We, J. H.; Shin, J. S.; Lee, H. E.; Oh, M.-W.; Lee, K. J.; Cho, B. J., Post ionized defect engineering of the screen-printed Bi2Te2. 7Se0. 3 thick film for high performance flexible thermoelectric generator. Nano Energy 2017,31, 258-263.

[110] Kim, C. S.; Lee, G. S.; Choi, H.; Kim, Y. J.; Yang, H. M.; Lim, S. H.; Lee, S.-G.; Cho, B. J., Structural design of a flexible thermoelectric power generator for wearable applications. Appl. Energy 2018,214, 131-138.

[111] Oh, J. Y.; Lee, J. H.; Han, S. W.; Chae, S. S.; Bae, E. J.; Kang, Y. H.; Choi, W. J.; Cho, S. Y.; Lee, J.-O.; Baik, H. K.; II, L. T., Chemically exfoliated transition metal dichalcogenide nanosheet-based wearable thermoelectric generators. Energy Environ. Sci. 2016,9, 1696-1705.

[112] Cai, X.; Sushkov, A. B.; Suess, R. J.; Jadidi, M. M.; Jenkins, G. S.; Nyakiti, L. O.; Myers-Ward, R. L.; Li, S.; Yan, J.; Gaskill, D. K.; E., M. T.; Dennis, D. H.; S., F. M., Sensitive room-temperature terahertz detection via the photothermoelectric effect in graphene. Nat. Nanotechnol. 2014,9, 814.

[113] Muench, J. E.; Ruocco, A.; Giambra, M. A.; Miseikis, V.; Zhang, D.; Wang, J.; Watson, H. F.; Park, G. C.; Akhavan, S.; Sorianello, V.; Michele, M.; Andrea, T.; Camilla, C.; Marco, R.; C., F. A.; Ilya, G., Waveguide-integrated, plasmonic enhanced graphene photodetectors. Nano Lett. 2019,19, 7632-7644. 